creams and the judicious use of the anti-viral drug "acyclovir" which can be used prophylacticly although more usually is applied when the first tingling symptoms or signs of rash appear. For treatment "acyclovir" can be applied to lesions or systemically in the more severe forms of infection.

\section{Hepatitis B}

This infection should not be confused with hepatitis $A$ which is spread via the faecal-oral route. Hepatitis $B$ is contracted almost exclusively through contact with infected blood or blood products, or sexually. The death rate from the acute infection is less than $1 \%$, with most cases becoming "non-infections" in 3 to 6 months. In contrast those who become carriers initially usually have an asymptomatic illness, thus making it difficult to know which individuals should be taking special care to avoid transmitting infection.

The tendency in recent years has been to improve overall hygiene precautions rather than to concentrate upon those known to be carrying the virus. Any injuries, cuts or grazes that bleed are potentially a source of infection if the blood comes in contact with open wounds of another person. The virus can survive outside the body for some hours, depending upon environmental circumstances and this can lead to infection for example through the sharing of toothbrushes or razors.

Transmission through bleeding into communal bath water theoretically may occur. Both dilution and the presence of chlorine will reduce this risk although showering is undoubtedly safer, and more hygienic! The risk of spread through sporting activities themselves must be small although reports have been made of cases possibly due to blood transfer between the feet of barefoot cross-country runners or via contaminated thorn-pricks during orienteering events. When people are known to have been exposed or may knowingly become exposed, immunisation either passive or active or a combination of both is available. This may perhaps be considered for certain types of athletes competing in countries known to have a high carriage rate of virus among the local populations.

\section{Acquired Immune Deficiency Syndrome (AIDS)}

Routes of transmission of human immunodeficiency virus (HIV) are almost identical to those of the hepatitis B virus. Persons with HIV are probably most infectious shortly after the original exposure and again when the symptoms of AIDS are beginning to appear. It seems probable from the evidence available that the infection is more difficult to transmit by needlestick injuries than hepatitis B. Transfer of infection within a household, unless factors such as sexual exposure or drug abuse have been involved, are very rare (Friedland et al, 1986). Similarly the spread of infection through normal sporting activities must be unusual. As with hepatitis $B$, detecting the asymptomatic carrier can be virtually impossible and an awareness of the need for overall good hygiene especially when dealing with wounds and care to avoid sexual exposure, are essential.

Herpes, hepatitis and AIDS are all more or less avoidable, depending very largely on personal behaviour and practices. Herpes is the most highly infectious, but poses the least threat to life. In contrast AIDS would appear to be invariably fatal, but does not spread readily from person to person and scarcely at all amongst those leading a normal life-style (Sharp, 1987).

\section{References}

Friedland, G. H., Saltzman, B. R., Rogers, M. F. Cahl, P. A., Lesser, M. L. Mayers, M. M. and Klein, R. S., 1986 "Lack of transmission of HTLV-III/LAV infection to household contacts of patients with AIDS or AIDS related complex with oral candidiasis". N.Engl.J.Med. 314: 344-9.

Sharp, J. C. M., 1987 "Virus infections in sport: herpes, hepatitis B and AIDS". Sports Update Medical Sciences No. 8, May 1987.

\section{M.E. - POST VIRAL FATIGUE SYNDROME \\ Dr. K. E. FEGAN, MRCGP, DObstRCOG \\ General Practitioner, West Kilbride, Ayrshire}

Myalgic encephalomyelitis (ME) is the latest name to encompass a syndrome complex that involves all or part of the muscles, the brain and its myelin covering. Post-viral fatigue syndrome suggests a possible viral cause.

As an illness ME occurs spectacularly more often in females in outbreaks, although it is otherwise evenly distributed between the sexes (Fegan et al, 1983). It has a predilection for age groups $25-35$ and is more noticed in the social classes I and II. The symptoms presented can be enormously varied. Muscle symptoms can be pain on exertion, twitching or just simple rapid and excessive fatigue. The central nervous symptoms initially consist of vertigo with associated sleep disturbance, nightmares, lack of concentration, occasional dysphasia, memory loss, mood disturbance and severe anxiety states; other symptoms can vary with paraesthesia and hyperacusis. Other features appear to be a cyclical and prolonged convalescence with only $60 \%$ of affected patients being better after two years.

The primary contact in an illness is usually the general practitioner but equally may well be that of the coach, physiotherapist or team manager. From this unique position and knowledge of individuals they can spot change. The diagnosis however is an exclusion diagnosis often made retrospectively after at least a period of six months.

Positive findings include partial hypogammaglobulinaemia, depressed IgG secretion by lympocytes, reduced helper/suppressor cell ratio, altered immune suppression, occasional absolute and atypical lymphocytosis. Other findings are abnormal muscle metabolism with increased lactic acid, while $75 \%$ of biopsies show muscle fibre necrosis and neuro-physiological abnormalities (Arnold et al, 1984, Jamal and Hensen, 1985). Aetiology appears to be related to the immune response to infection with differing viruses producing different end-organ effects and the concept of a chronic viral illness must now be recognised (Yousef et al, 1988). Various viruses (Behan et al, 1985) have been incriminated including the Coxsackie group, Epstein-Barr, varicella, influenza and measles viruses. The Coxsackie virus in particular appears to be incriminated and attracts attention as it is known to be both myotropic and neurotropic and there are numerous anecdotal cases of sudden death in sportsmen during exercise (Roberts, 1986) with the Coxsackie virus being recovered post-mortem from myocardial tissue.

The illness seems to affect high achievers and the link between stress and susceptibility to infection has always been of interest. 
Treatment must initially be rest especially during the acute phase. Avoidance of caffeine, alcohol, aspirin and activities may well be of benefit and the use of anxiolytics, antidepressants and analgesics is important but should be considered only if absolutely necessary. Such a long illness produces conditioning and habit-forming. This restricted advice is often too minimal for most patients and the tendency is to self-refer elsewhere or self-medicate with exclusion diets, dietary supplements and allergy treatments - only anecdotal evidence is available as to their benefits.

Considering prevention, it seems prudent to avoid exercise during any active viral illness and the time to return to training to be 2-4 weeks following an infection associated with pyrexia and/or involvement of the lymphoid system. Energetic self-help groups have evolved with vast numbers of members, who contribute to the funding of research. Several well known sports personalities have succumbed to this disorder, whose careers have been devastated. David Provan a professional footballer with Glasgow Celtic FC gave a vivid account of his personal experience of the illness. When feeling unwell he would train harder, but this was the worst possible thing to have done, and even now can only walk a few hundred yards before becoming fatigued.

The somatic presentation and subsequent management of depression, anxiety, hysteria are not helped by a medical model as vague as "post-viral fatigue syndrome" when the initial psychological conflicts are left unresolved. The role of the general practitioner is therefore crucial in spotting change, particularly with his knowledge of the previous personality of the individual, but along with the coach and team manager must be wary of "Heightened medical awareness" and the creation of pseudo-epidemics.

The true post-viral fatigue syndrome patient suffers a long, isolated illness, deserving of maximum sympathy and support. The reason for their vulnerability and susceptibility to this disorder remains as yet unclear.

\section{References}

Arnold, D. L., Bore, P. J., Radda, G. K., Styles, P. and Taylor, D. J., 1984 "Excessive intra-cellular acidosis of skeletal muscle on exercise in a patient with post-viral exhaustion syndrome". The Lancet I: 1367-1369.

Behan, P. O., Behan, W. M. H. and Bell, E. J., 1985 "The post-viral fatigue syndrome - analysis of the findings: 50 cases". J. of Infect. 10: 211-222.

Fegan, K. G., Behan, P. O. and Bell, E. J., 1983 "Myalgic encephalomyelitis". Report of an epidemic". J.Roy.College General Practitioners 33: 335-337.

Jamal, G. A. and Hensen, S., 1985 "Electrophysiological studies in the postviral fatigue syndrome". J.Neurology, Neurosurgery and Psychiatry 48: 691-694.

Roberts, J. A., 1986 "Viral illness and sports performance". Sports Medicine 3: 296-303.

Yousef, G. E., Bell, E. J., Mann, G. F., Murugesan, V., Smith, D. G., McCartney, R. A. and Mowbray, J. F., 1988 "Chronic entero-virus infection in patients with post-viral fatigue syndrome". The Lancet I: 146-149.

\section{TRAVELING AND TOURING PROBLEMS}

\section{J. H. COSSAR, MD}

General Practitioner \& Research Associate, Communicable Diseases (Scotland) Unit, Ruchill Hospital, Glasgow

The importance of infection by travellers has been recognised since before 1377 when ships arriving into ports of the
Venetian Republic were kept at a distance and travellers detained in isolation for $\mathbf{4 0}$ days (quaranta giorni) before they were allowed to proceed to their final destination the origin of the concept of quarantine.

The Scot has an historical tradition of travel abroad and this prompted an enquiry into the health hazards experienced by Scottish Presbyterian missionaries who served abroad between 1867 and 1929. Overall of the 1416 missionaries studied, 20 per cent returned prematurely due to ill health, five per cent due to family ill health and 151 (11 per cent) died in service; 36 per cent of the deaths were caused by infection (18 per cent due to malaria). Later appointment (post-1900) was associated with fewer retirements due to ill health and greater longevity in missionaries. The effect of geographic location was marked by higher morbidity and mortality rates for those serving in tropical Africa; Calabar in West Africa had the highest rates.

The numbers of international travellers continue to rise annually, particularly so over the last 20 years, and included in this group are those participating in sporting events at both club and national level.

A facility has been developed at the Communicable Diseases (Scotland) Unit (CD(S)U) whereby the health of returning Scottish travellers can be monitored and specific enquiry carried out on groups identified as being "at risk" from a health hazard whilst abroad. Between 1793 and 1985 , out of 14,227 study respondents, 37 per cent gave a history of illness while overseas. Alimentary symptoms predominated; 18 per cent had these alone and a further 10 per cent had other symptoms associated with their gastro-intestinal disorder. Higher attack rates were associated with package holidays, inexperience of travel, smoking, summer travel, travel further south and east, and younger age (particularly those aged 20-29 years - which age group includes the majority of athletes). During 1973 for example, four members of a Scottish ladies football team touring in Morocco developed paratyphoid fever.

Serological studies on $\mathbf{4 7 0}$ of the travellers showed that 20 per cent had incomplete immunity to poliomyelitis. Less than two per cent (of 760 tested) had antibodies to Legionella pneumophila and in $\mathbf{5 1 1}$ travellers seropositivity to hepatitis $A$ ranged from 30 per cent (those aged 20 years) to 89 per cent (those aged 60 years).

Other international studies of illness in returning travellers, show that younger age groups (specifically those aged from 20-29 years) and those experiencing the greatest climatic and cultural contrast to their home country are most at risk, with attack rates of up to $\mathbf{4 0}$ per cent (Steffen et al, 1983; Peltola et al, 1983).

Analysis of laboratory reports on infections among travellers, in particular Salmonellosis, bacillary dysentery and campylobacter enteritis, collated at the $C D(S) U$ show a five-fold increase in the annual total between 1975 and 1986 and an increase in the proportion of isolates of pathogens from holidaymakers (from 62 to 90 per cent of the total) during the same period. In addition admissions to the infectious diseases wards at Ruchill Hospital, Glasgow during 1985 show that 6 per cent of the 1,265 admissions were travel associated. Travel to the Indian subcontinent was associated with 60 per cent of the admissions, with the 20-29 year age group once more having the highest representation (25 per cent); malaria was the largest single diagnosis (37 per cent of the total).

The "at risk" profile that emerges suggests that the sportsman and sportswoman travelling abroad would be 